\title{
Molecular Mechanisms of Resistance to Conventional Antibiotics in Bacteria
}

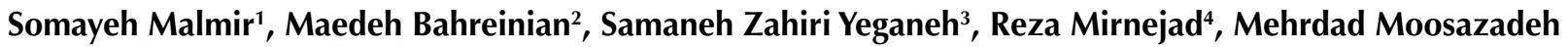 \\ Moghaddam $^{5^{*}}$, Fatemeh Saberi ${ }^{6^{*}}$ \\ ${ }^{1}$ Nanobiotechnology Research Center, Baqiyatallah University of Medical Sciences, Tehran, Iran \\ ${ }^{2}$ Department of Molecular Genetics, Research Center for Biosciences and Biotechnology, Malek Ashtar University, Tehran, Iran \\ ${ }^{3}$ Department of Microbiology, Faculty of Sciences, Karaj Branch, Islamic Azad University, Karaj, Iran \\ ${ }^{4}$ Molecular Biology Research Center, Systems Biology and Poisonings Institute, Baqiyatallah University of Medical Sciences, Tehran, \\ Iran \\ ${ }^{5}$ Applied Biotechnology Research Center, Baqiyatallah University of Medical Sciences, Tehran, Iran \\ ${ }^{6}$ Department of Biotechnology, Student Research Committee, School of Advanced Technologies in Medicine, Shahid Beheshti \\ University of Medical Sciences, Tehran, Iran
}

Corresponding Authors: 1. Mehrdad Moosazadeh Moghaddam, Applied Biotechnology Research Center, Baqiyatallah University of Medical Sciences, Tehran, Iran. Email: mm.genetics@gmail.com

2. Fatemeh Saberi, Department of Biotechnology, Student Research Committee, School of Advanced Technologies in Medicine,

Shahid Beheshti University of Medical Sciences, Tehran, Iran. Email: fatemeh.saberi@sbmu.ac.ir

Received April 18, 2018; Accepted June 27, 2018; Online Published September 30, 2018

\begin{abstract}
In the early years of the 20th century, the medical world was able to discover drugs that can eliminate microbial infections, and in the mid-twentieth century gradually began the clinical application of these drugs as antibiotics. Soon, however, scientists found that some microbes become resistant to these drugs and began attempts to identify new antibiotics. At the same time, microbes were also considering changes to escape the effects of antibiotics. The reality is that, like any other living creature, microorganisms, especially bacteria, adapt to their surroundings in order to survive. Therefore, the bacteria that were already affected by one or more antibiotics became resistant to them. Classically, drug resistance in bacteria is attributed to chromosomal mutations, but chiefly, it seems that it is associated with extrachromosomal elements acquired from environmental bacteria. Accordingly, this review investigated the molecular mechanisms that lead to drug resistance in bacteria.

Keywords: Antibiotic, Drug resistance, Bacteria, Molecular mechanisms

Citation: Malmir S, Bahreinian M, Zahiri Yeganeh S, et al. Molecular mechanisms of resistance to conventional antibiotics in bacteria. Int J Med Rev. 2018;5(3):118-129. doi:10.29252/JJMR-050305.
\end{abstract}

\section{Introduction}

The first antibiotic was discovered in 1928 by Alexander Fleming, who accidentally noticed the antibacterial effect of a substance released by Penicillium notatum. Later, Howard Walter Florey purified this substance and administered it to patients to treat infections systemically. Subsequently, scientists identified other natural substances that could be produced as semi-synthetic antibiotics after their structures were altered. Finally, new and fully synthetic antibiotics were manufactured with greater efficacy and more chemical stability (Figure 1). Meanwhile, drug resistance was emerging even in organisms encountering the newly-made antibiotics for the first time (Figure 2). The most notable example is the emergence of penicillin resistance among staphylococci, caused by an enzyme (penicillinase) which inactivates this antibiotic. Generally, with the discovery and presentation of various antibiotics over many years, continued drug-induced selection pressure has led to the growth and development of microorganisms containing various types of resistance mechanisms, resulting in multidrug resistance (MDR). Some of these mechanisms include the production of penicillinbinding proteins (PBPs), enzymatic mechanisms of drug modification, mutated drug targets, enhanced efflux pump expression, and altered membrane permeability (Figure 3). Some of the most problematic MDR microorganisms include Pseudomonas aeruginosa, Acinetobacter baumannii, Escherichia coli, Klebsiella pneumonia, extended-spectrum $\beta$-lactamases (ESBL), vancomycin-resistant enterococci (VRE), methicillin-resistant Staphylococcus aureus, and extensively drug-resistant Mycobacterium tuberculosis. ${ }^{1-4}$ This article is aimed to review the routine genetic and molecular mechanisms of resistance to conventional antibiotics, rather than providing a broad list of antibiotic resistance mechanisms that can be found in numerous articles, which are related to

Copyright (C) 2018 The Author(s). This is an open-access article distributed under the terms of the Creative Commons Attribution License (http:// creativecommons.org/licenses/by/4.0), which permits unrestricted use, distribution, and reproduction in any medium, provided the original work is properly cited. 


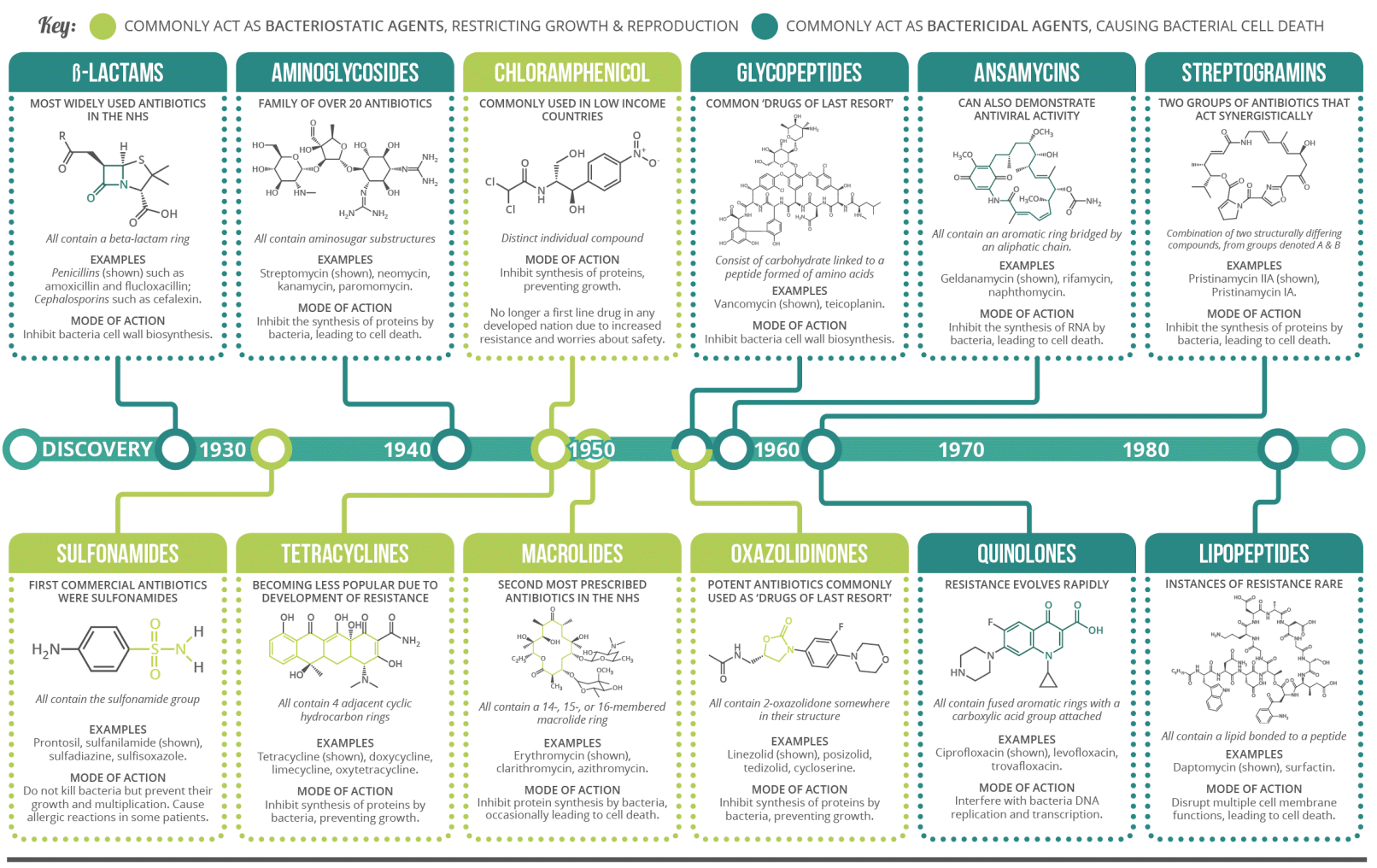

Figure 1. Different antibiotic classes at a glance (https://www.compoundchem.com/2014/09/08/)

important pathogens and drugs used by physicians today.

\section{Genetics of Multidrug Resistance in Microorganisms}

Bacterial antibiotic resistance can be achieved through intrinsic or acquired mechanisms (Figure 3). Intrinsic mechanisms are those specified by naturally occurring genes found in the chromosomal structure of the cell, such as AmpC $\beta$-lactamase in gram-negative bacteria, and many of the efflux systems in the cell membrane. Acquired mechanisms are characterized by factors such as mutations in genes targeted by the antibiotic and the transfer of resistance determinants borne on plasmids, bacteriophages, transposons, and other mobile genetic materials (Table 1). ${ }^{3}$ In general, this exchange is accomplished through the processes of transduction (by bacteriophages), conjugation (by plasmids and conjugative transposons), and transformation (through incorporation into the chromosome of DNA, plasmids, and other DNAs from dying organisms). Although gene transfer is common among microorganisms within the same genus, this process has been observed among very different generations, including such environmentally distant organisms as gram-positive and gram-negative bacteria. Plasmids contain genes for resistance and many other traits; they replicate independently of the host chromosome and can be distinguished by their origins of replication. Multiple plasmids can exist within a bacterium, and their genes can be added to the genetic integrity of the microorganism. Transposons are mobile genetic elements that can exist on plasmids or integrate into other transposons or the host's chromosome. In general, these pieces of DNA contain terminal regions that participate in recombination and specify a protein (such as transposase or recombinase), which facilitates incorporation into and out of specific genomic regions. Integrons contain collections of genes (gene cassettes) that are generally classified according to the sequence of the protein (integrase) that participate in their recombination function. They have the ability to integrate stably into regions of other DNAs, where they deliver, in a single exchange, multiple new genes, particularly drug resistance. The super-integron, which consists of hundreds of genetic cassettes (accounting for about 3\% of the host genome), differs from other integrons. These elements were first detected in Vibrio cholera. ${ }^{6-8}$

\section{Mechanisms of Intrinsic Antibiotic Resistance \\ 1. Resistance Through Chromosomal Mutation Fluoroquinolones}

Almost all important fluoroquinolone resistance can be attributed to mutations within the drug's targets, DNA gyrase and topoisomerase IV. These complex molecules perform critical ATP-dependent functions during DNA replication. Each is comprised of several subunits: GyrA and GyrB for DNA gyrase and ParC/GrlA and ParE/GrlB for topoisomerase IV. The GyrA and ParC/GrlA proteins contain the DNAbinding functions and are targeted by fluoroquinolones (which are purely synthetic antibiotics), whereas GyrB and ParE/GrlB play the roles of ATP binding and hydrolysis and are inhibited by coumarin antibiotics. As the first step in developing resistance to fluoroquinolone, mutations occur in the DNA gyrase of the gram-negative bacteria, whereas the initial mutations occurring in the topoisomerase IV of the gram-positive bacteria result in initial resistance. Mutations that lead to fluoroquinolone resistance are found mainly in 
A Timeline of Key Events ${ }^{\text {}}$

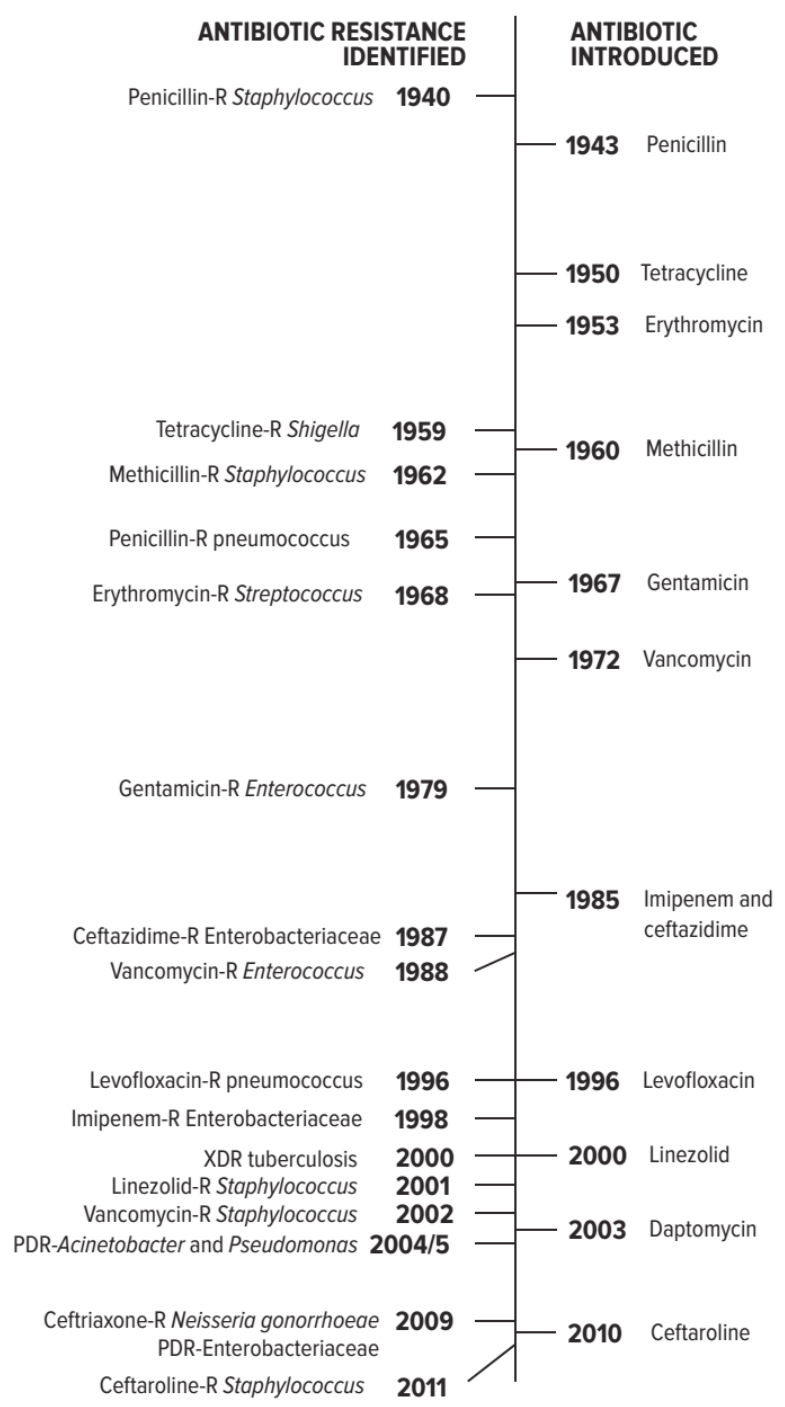

Figure 2. Timeline for the Introduction of Conventional Antibiotics as well as the Time of Appearance of the First Bacterial Resistance. ${ }^{5}$ (PDR = Pan-Drug Resistant; $\mathrm{R}=$ Resistant; XDR = Extensively Drug-Resistant).

the quinolone resistance-determining region (QRDR) of GyrA and ParC/GrlA. In general, bacterial isolates that are highly resistant to fluoroquinolone carry multiple QRDR mutations as well as other mechanisms for fluoroquinolone resistance such as drug efflux pumps. ${ }^{9-12}$

\section{Rifampicin}

Rifampicin is a bacterial drug that stops transcription by interacting with RpoB in the RNA polymerase beta subunit. Although combination therapies including rifampin or rifapentine, isoniazid, pyrazinamide, ethambutol, and streptomycin, are still the primary choices for the treatment of $M$. tuberculosis-induced infections, significant drug resistance still occurs in the rifampin-binding region of rpoB through point mutations at a frequency of $1 \times 10^{-6} \cdot{ }^{13,14}$

\section{Sulfonamides and Trimethoprims}

Sulfonamides, which are the first antimicrobial compounds and were manufactured in large scales in 1935 for clinical

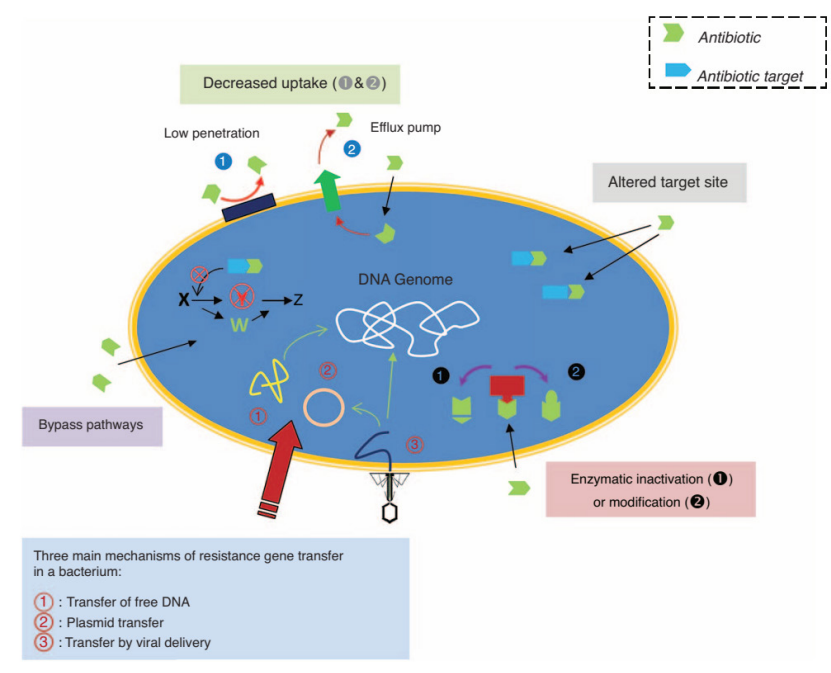

Figure 3. A Schematic View of the Mechanisms of Antimicrobial Resistance in Bacteria. Reprinted by permission from Wolters Kluwer Health, Inc: Reviews in Medical Microbiology. See the following link for details: https://journals.Iww.com/revmedmicrobiol/fulltext/2015/07000/The_ development_of_antimicrobial_peptides_as_an.4.aspx. ${ }^{3}$

administrations, target the dihydropteroate synthase. Trimethoprim, introduced in 1968, inhibits dihydrofolate reductase and was the last structurally unique antibiotic approved prior to the release of linezolid in 2000. Mutations in the gene specifying dihydropteroate synthase reduce the binding affinity of the enzyme to sulfonamides, and have been found in laboratory samples of E. coli and Streptococcus pneumonia as well as in clinical isolates of Campylobacter jejuni and Haemophilus influenzae. Mutations in the gene specifying dihydrofolate reductase can result in the over-expression of an enzyme with a reduced binding affinity for trimethoprim, inducing a high degree of resistance to trimethoprim in E. coli and $H$. influenzae..$^{15-17}$

Tetracycline, Aminoglycoside, and Macrolide-LincosamideStreptogramin Antibiotics

Antibacterial agents in tetracycline, aminoglycoside, and macrolide-lincosamide-streptogramin (MLS) classes target ribosomes in order to inhibit the translation of RNA into proteins; that is why chromosomal resistance through chromosomal mutation is not common. Tetracyclines and aminoglycosides interact with 16S rRNA (rrs), and the MLS family bind to $23 \mathrm{~S}$ rRNA ( $\mathrm{rrl}$ ). In most bacteria, multiple rrs and rrl operons are present, and susceptibility caused by each of these targets can be dominant, making resistance difficult to achieve without a mutation in all or a majority of the other operons. However, in organisms with low rRNA (rrn) copy numbers, chromosomal mutations that cause resistance have appeared. Tetracycline resistance caused by a point mutation in Propionibacterium acnes ( 3 rRNA operons) and Helicobacter pylori (2 copies of rrn) has been recorded. Mutations in rrs lead to resistance to amikacin and kanamycin and alterations in small ribosome protein S12 (rpsL) or rrs affecting streptomycin (all aminoglycoside drugs) susceptibility in clinical $M$. tuberculosis (1 rrn operon) have been reported. The emergence of resistance 
Table 1. Major Genetic Elements in Transfer of Drug Resistance Genes

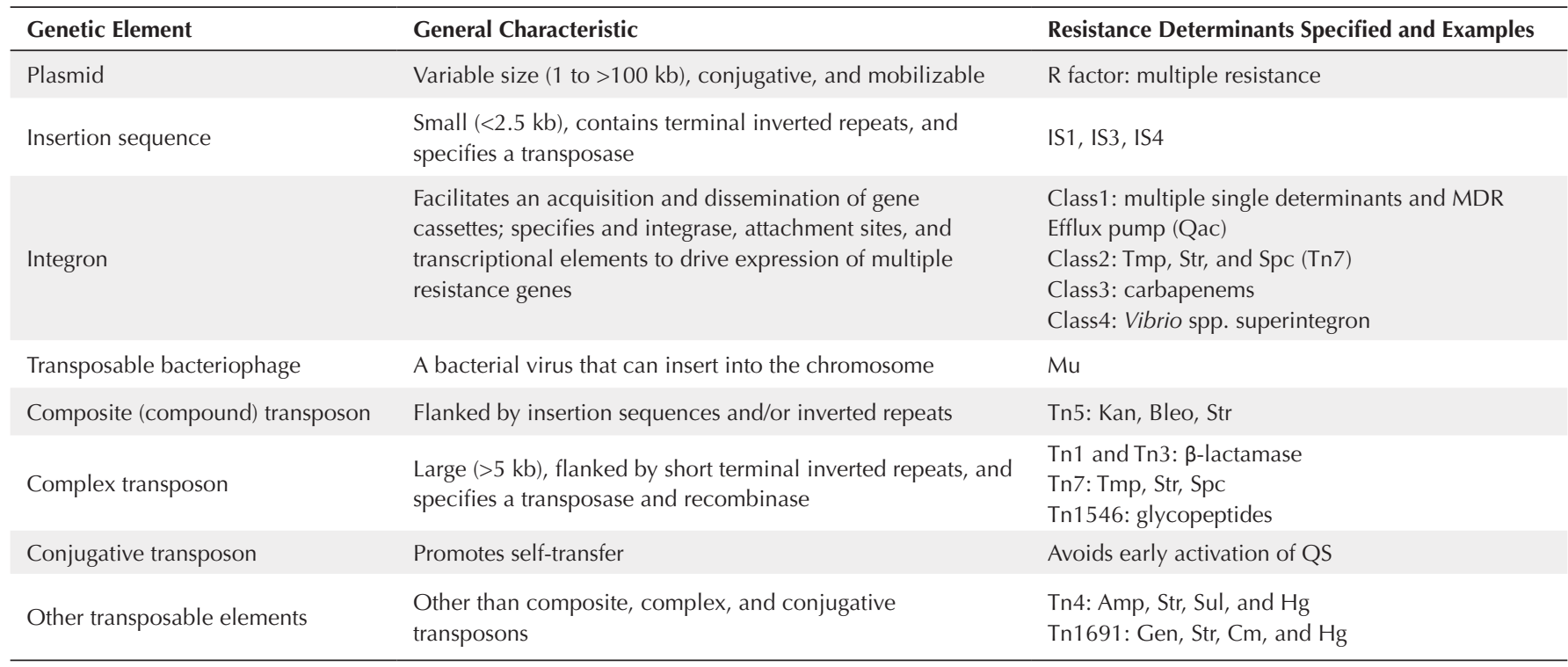

to erythromycin (a macrolide) caused by mutant $\mathrm{rrl}$ in $S$. pneumonia (4 copies of rrn) has also been reported. Moreover, mutations in the large ribosome protein L4 (rplD) have also been shown to alter MLS susceptibility. Ketolides, introduced into clinical applications 2 decades ago, are designed to reduce macrolide resistance; nonetheless decreased resistance to telithromycin (a ketolide) has been found in S. pneumonia with mutations in $\mathrm{rrl}, \mathrm{rplD}$, and large ribosome protein L22 (rplV). Previous studies have shown that mutations in L22 also affect quinupristin-dalfopristin (streptogramins that act individually in a bacteriostatic manner) susceptibility by affecting their synergistic relationship, which is of high importance to the combination's bactericidal mechanism of action. ${ }^{18-22}$

\section{Oxazolidinones}

Linezolid (another inhibitor of protein production) has been approved as an agent to treat methicillin-resistant $S$. aureus and vancomycin-resistant enterococci (VRE) infections. The availability of both intravenous and oral formulations of these antibiotics makes them applicable for use both in- and out-of-hospital settings. Resistance to linezolid in laboratory studies has been related to point mutations in $\mathrm{rrl}$ in $S$. aureus and Enterococcus faecalis. Linezolid-resistant clinical isolates of Staphylococcus epidermidis, S. aureus, Streptococcus oralis, Enterococcus faecium, and E. faecalis have been confirmed and documented, and many of them bear rrl mutations. Similar to fluoroquinolones, the level of resistance in S. aureus increases extensively with mutations in multiple rrl alleles.

\section{Lipopeptides}

Daptomycin (a drug acting on bacterial membranes) was approved by the Food and Drug Administration (FDA) in 2003. Although it has been successfully used for treating infections caused by bacteria, treatment failures have also been reported. Studies have shown that mutations in multiple chromosomal loci (e.g., mprF, yycG, rpoB, and rpoC) affect daptomycin susceptibility. ${ }^{27-29}$

\section{Resistance Through Genomic Duplications}

Another common mechanism for drug resistance is gene amplification resistance, which leads to the overexpression of multidrug transporters and drug targets. For example, largescale duplications of the acrAB locus of the mutant bacteria E. coli have been detected in the presence of tetracycline, and it results in the over-expression of the acrAB efflux pump, creating a kind of bacterial MDR phenotype. The mutants, however, are unstable and revert to the wild-type phenotype in the absence of the drug. Previous studies have also shown that genomic amplification affects susceptibility to methicillin in $S$. aureus. It is expected that the use of gene duplication as a mechanism of resistance is increasing among bacterial isolates. However, in this case, the likely phenotype will be an unstable form of resistance (Table 2)..$^{18,30,31}$

\section{Mechanism of Acquired Antibiotic Resistance \\ 1- Enzymatic Drug Modification}

Enzymes that modify antibacterial drugs are divided into 2 general classes: those such as $\beta$-lactamases that degrade antibiotics, and others that perform chemical transformations (including the macrolide and aminoglycoside-modifying proteins). ${ }^{33}$

\section{$\beta$-Lactam Antibiotics}

There are hundreds of $\beta$-lactamases. Most resistance is caused by genes located on plasmids and transposons; others are chromosomal and provide intrinsic resistance. $\beta$-lactamases are classified using indicators based on function (the system of Bush-Jacoby-Medeiros) or structure (Ambler classification), but in general, they are broadly divided into enzymes with a serine in the active site and those that require a metal ion cofactor. The Ambler classification system divides 
Table 2. Methods of Bacterial Drug Resistance and Relevant Target Antibiotics ${ }^{32}$

\begin{tabular}{|c|c|c|c|}
\hline Basis or Resistanc & Mechanism & $\begin{array}{l}\text { Bacterial Proteines/Target } \\
\text { Responsible }\end{array}$ & Antibiotic Target \\
\hline \multirow{3}{*}{ Enzyme } & Hydrolysis & $\begin{array}{l}\beta \text {-Lactamase } \\
\text { Esterase } \\
\text { C-P lyase complex }\end{array}$ & $\begin{array}{l}\beta \text {-Lactamase } \\
\text { Macrolide } \\
\text { Fosfomycin }\end{array}$ \\
\hline & Group transfer & $\begin{array}{l}\text { Acetytransferase } \\
\text { Phosphotransferase } \\
\text { Nucleotidyltransferase } \\
\text { glycosyltransferase } \\
\text { Ribosyletransferas } \\
\text { Thioltransferase }\end{array}$ & $\begin{array}{l}\text { Streptogramins, Aminoglycosides, } \\
\text { Chloramphenicol } \\
\text { Aminoglycosides, Macrolides } \\
\text { Lincomycin, Clindamycin, Aminoglycosides } \\
\text { Macrolid } \\
\text { Rifampin } \\
\text { Fosfomycin }\end{array}$ \\
\hline & Redoox process & tetX & Tetracyclines \\
\hline Target modification & $\begin{array}{l}\text { Structural } \\
\text { Alteration/ modifications } \\
\text { Mutationsin genes } \\
\text { Aminoacid substitutions } \\
\text { Methylation } \\
\text { Mutation }\end{array}$ & $\begin{array}{l}\text { Penicillin binding proteins } \\
\text { Cell wall precursors } \\
\text { Ribosomal subunits } \\
\text { RNA polymerase } \\
\text { DNA Gyrase/topoisomerase } \\
16 \mathrm{~S} \text { rRNA } \\
23 \mathrm{~S} \text { rRNA } \\
23 \mathrm{~S} \text { rRNA }\end{array}$ & $\begin{array}{l}\text { b lactam antibiotics } \\
\text { vancomycin } \\
\text { streptomycin } \\
\text { rifamycines } \\
\text { quinolones } \\
\text { aminoglycosides } \\
\text { Macrolide } \\
\text { Oxazolidinones }\end{array}$ \\
\hline Reduced permeability & Reduced protein/expression/defective & Porins & $\begin{array}{l}\text { B-lactams/fluoroquinolones/aminoglycosides/ } \\
\text { chloramphenicol }\end{array}$ \\
\hline Target protection & Ribosom protection & Ribosome protection proteins & Tetracycline \\
\hline Efflux & Active extrusion & Membrane proteins & all major antibiotics \\
\hline
\end{tabular}

$\beta$-lactamases into four groups: class $\mathrm{A}, \mathrm{C}$, and D enzymes are proteins with a serine amino acid at their active sites, and class $\mathrm{B}$ proteins are zinc-dependent metalloenzymes. Some class A proteins function as ESBL and as carbapenemases. Class B metalloenzymes that hydrolyze carbapenems are susceptible to inhibition by EDTA, but, in contrast, not susceptible to inhibition by clavulanate (a $\beta$-lactamase inhibitor). AmpC, an inducible and usually chromosomal enzyme found in many species of the Enterobacteriaceae and P. aeruginosa, is a prototype of the class $\mathrm{C}$ enzyme. Recent reports have shown plasmid-borne ampC genes that can be transferred among $E$. coli, Klebsiella spp., and Salmonella species. Class D enzymes have been found in only a few species such as P. aeruginosa, Acinetobacter, and Aeromonas (Figure 4). ${ }^{34-36}$

\section{Aminoglycosides}

A large number of aminoglycoside-modifying enzymes are produced by genes located on transferable elements. This type of resistance is accomplished with proteins that $\mathrm{N}$-acetylate (acetyltransferase), phosphorylate (phosphotransferase), and adenylate (nucleotidyltransferase) aminoglycosides. The acetyltransferases are able to modify tobramycin, gentamicin, netilmicin, and amikacin; the nucleotidyltransferase proteins alter the activity of tobramycin; and the phosphotransferases affect amikacin susceptibility. Many of the aminoglycosidemodifying enzymes are found on integrons and other mobile genetic elements. For example, three acetyltransferase genes were found on a class 1 integron from $P$. aeruginosa that causes resistance to carbapenems and sulfonamides. ${ }^{37-39}$

\section{Macrolide-Lincosamide-Streptogramin Antibiotics}

There are a number of inactivating enzymes that affect MLS antibiotics. Their genes encode esterases, hydrolases, glycosylases, phosphotransferases, nucleotidyltransferases, and acetyltransferases and are found less frequently than efflux and ribosome-modifying genes in clinical isolates. Esterases act on 14- (e.g., erythromycin) and 15- (e.g., azithromycin) membered macrolides; the hydrolases affect streptogramin B drugs. Acetyltransferases inactivate streptogramin A antibiotics, and nucleotidyltransferases produce resistance to lincosamides (e.g., clindamycin). Phosphotransferases modify 14-, 15-, and 16-membered macrolides by modifying their characteristics. ${ }^{40-41}$

\section{Chloramphenicol}

The acetyltransferases that inactivate chloramphenicol are the most common resistance mechanisms for these antibiotics and are divided into two types, A and B enzymes, both of which act as homotrimers but are not related based on amino acid sequence analyses. The type B enzymes are also termed xenobiotic acetyltransferases and seem to share an evolutionary lineage that includes some streptogramin-inactivating enzymes found in enterococci and staphylococci. ${ }^{41,42}$

\section{Tetracyclines}

A flavin-dependent monooxygenase, designated tet(X), has been identified in Bacteroides fragilis that acts on older tetracyclines (such as tetracycline, oxytetracycline, and chlortetracycline) as well as newer compounds (such as doxycycline, minocycline, and tigecycline). This enzyme catalyzes regioselective hydroxylation to inactivate its initial target, but the products of this enzyme are unstable at a physiological $\mathrm{PH}$. Although a related tet(X)-like gene in $P$. aeruginosa has been reported, the presence and interference of this gene have not been reported in tetracycline-resistant clinical isolates. ${ }^{43,44}$

\section{Altered, Substituted, and Protected Drug Targets $\beta$-Lactam Antibiotics}

The first penicillin-resistant S. aureus, identified in the mid- 


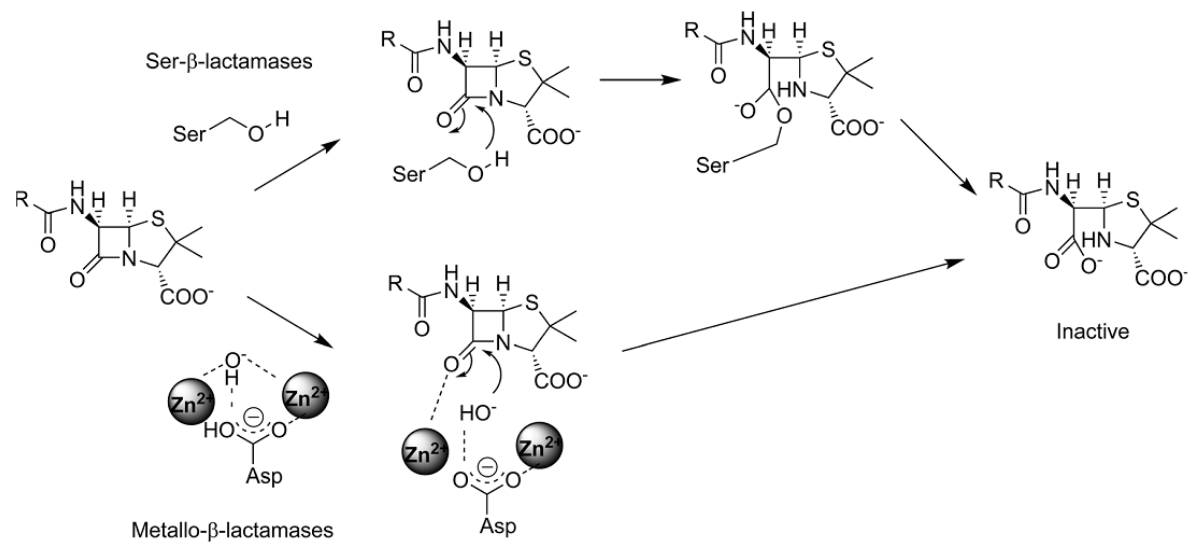

Figure 4. Function of Serine and Metallo- $\beta$-Lactamases. Reproduced by permission of The Royal Society of Chemistry. See the following link for details: https://pubs.rsc.org/en/Content/ArticleLanding/2011/CC/c0cc05111j\#!divAbstract. ${ }^{36}$

1940s, expressed a $\beta$-lactamase (named PCI). Subsequently, methicillin, as a penicillin derivative which was resistant to this $\beta$-lactamase, was introduced in 1959 to treat penicillinresistant isolates. Methicillin-resistant $S$. aureus was then identified in 1961. The $\beta$-lactam resistance in these samples was linked to the acquisition of a gene capable of producing an altered PBP. In general, resistance frequently occurs in staphylococci and streptococci following the acquisition of genes encoding PBPs that are not sensitive to $\beta$-lactam inhibition. The altered PBP of methicillin-resistant $S$. aureus, PBP2a, is created by mecA and transported on a mobile genetic element called the "staphylococcal cassette chromosome" (SCCmec). In addition to mecA, SCCmec contains the mecR1-mecI regulatory loci and encodes enzymes that are involved in site-specific recombination. S. aureus uses multiple PBPs during cell wall biosynthesis under normal circumstances. One of them, PBP2, is a bifunctional enzyme with transpeptidase and transglycosylase activities. When methicillin-resistant $S$. aureus is exposed to methicillin, PBP2 acts as the transglycosylase, while using its transpeptidase activity to produce resistance to nearly all $\beta$-lactam antibiotics. Removal of the transglycosylase function of PBP2 leads to $\beta$-lactam susceptibility and demonstrates the importance of both functions of the enzyme. ${ }^{45}$

\section{Glycopeptides}

Glycopeptides (including vancomycin and teicoplanin) interact with bacterial peptidoglycan precursors. Resistance to glycopeptides in gram-positive cocci is another example of an altered drug target. In enterococci, acquired glycopeptide resistance is a trait attributable to VanA, B, $\mathrm{D}, \mathrm{E}$, and $\mathrm{G}$ phenotypes, while VanC is responsible for the intrinsic resistance. VanA and VanD cause resistance to both vancomycin and teicoplanin, whereas the others produce resistance to vancomycin alone. The resistance phenotype is accomplished using multiple proteins produced by gene clusters and each result in the production of a modified peptidoglycan. Of the many drug-resistance indexes recently known, the glycopeptide resistance inductor is probably the most complex one. ${ }^{47}$
The activity of many enzymes produced by the gene cluster is involved in causing glycopeptide resistance. Both a racemase and a dehydrogenase can result in the production of serine (VanC, E, or G) or lactate from pyruvate (VanA, B, or D), which a ligase uses to form a C-terminal D-Ala-D-Ser or D-Ala-D-Lac in the altered peptidoglycan (Figure 5). A two-component regulatory system controls the expression of the biosynthetic machinery. Although glycopeptides have a lower affinity to D-Ala-D-Ser or D-Ala-D-Lac, they can still bind and inhibit peptidoglycan biosynthesis if a D-Ala target remains intact. Two additional enzymes (or a single bi-functional protein for VanC) complete the phenotype by removing the normal target of the antibiotic: a dipeptidase cleaves the C-terminal D-Ala-D-Ala and a carboxypeptidase provides the redundant function of removing the terminal D-Ala in the absence of, or under circumstances where, this enzyme is less active. In recent years, the enterococcal vanA gene cluster has made its way into methicillin-resistant $S$. aureus and manifested an extensive vancomycin resistance. In all cases, the resistance elements are located on the plasmid of Tn1546 transposon. Vancomycin-resistant E. faecalis and methicillin-resistant $S$. aureus were also obtained from patients bearing vancomycin-resistant $S$. aureus, and each contained identical plasmids, except for the presence of Tn1546 in the isolate of vancomycin-resistant E. faecalis. It is assumed that the plasmid from E. faecalis was the vehicle for vanA entry into $S$. aureus and that the vanA gene cluster was subsequently transferred by means of transposition into the $S$. aureus plasmid (Figure 6)..$^{4-51}$

\section{Tetracyclines and MLS Antibiotics}

The most prevalent forms of resistance to tetracyclines in the clinic are drug efflux and ribosome protection. Ribosome protection factors have sequence similarity to bacterial elongation factors (EF-G an EF-Tu). They also possess GTPase activity and facilitate the release of tetracycline from the ribosome in a manner that requires energy. In Megasphaera elsdenii, a mosaic gene containing 2 ribosome protection factors (TetO and TetW) has been reported. Moreover, the tet $(\mathrm{P})$ element of Clostridium perfringens 


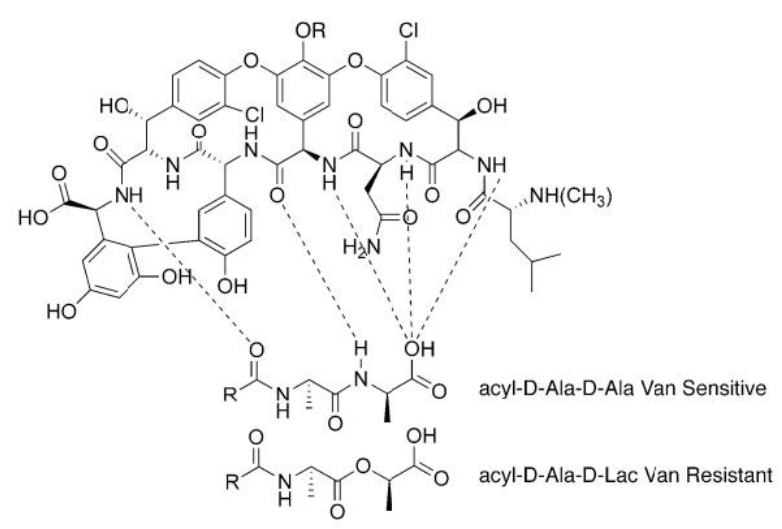

Figure 5. Vancomycin and other glycopeptides interact with the acyl-DAla-D-Ala portion of peptidoglycan using 5 hydrogen bridges. The figure shows a resistant type of an ester that replaces the amide group (acyl-D-AlaD-Lactate), and one of the hydrogen bonds is eliminated. Reproduced by permission of The Royal Society of Chemistry. See the following link for details: https://pubs.rsc.org/en/Content/ArticleLanding/2011/CC/ c0cc05111j\#! divAbstract. ${ }^{36}$

creates both ribosome protection and efflux mechanisms in an overlapping genetic unit. ${ }^{52}$ Drug binding within the MLSK (macrolide-lincosamide-streptogramin-ketolide) family to $23 \mathrm{~S}$ rRNA is affected by erm (erythromycin resistance methylase or erythromycin ribosome methylation) gene products. These mechanisms represent the predominant macrolide resistance mechanisms in Europe and South Africa. Currently, there are 34 different classes of Erm proteins (http://faculty.washington.edu/marilynr/), and each acts by methylating a single adenine in the E. coli $23 \mathrm{~S}$ rRNA (at position A2058). Methylation results in the demonstration of the MLSa phenotype, which causes resistance to 14-, 15-, and 16-membered lincosamides and streptogramin B. In staphylococci, agents like erythromycin and azithromycin induce erm expression, but 16-membered macrolides do not. Permanent erm expression in some clinical and laboratory strains causes telithromycin resistance..$^{53-55}$

\section{Sulfonamide and Trimethoprim Antibiotics}

The activities of the sulfonamides and trimethoprim are also affected by acquired genes specifying enzymes that are insensitive to drug inhibition. Sull and sul2 are the main elements of clinical resistance to sulfonamide, whereas sul3 was found to be prevalent in farm animals. In contrast, more than 20 trimethoprim resistance genetic elements (numbered chronologically from dfr1) have been documented. The genes specifying the sulfonamide-insensitive dihydropteroate synthases are present on class 1 integrons (sul1) or plasmids (sul2), whereas dfr variants (with dfr1 being the most common in gram-negative bacteria) move from bacterium to bacterium on class 1 and 2 integrons. The dfr 1 gene is located on the $\operatorname{Tn} 7$ transposon, facilitating its penetration into the $E$. coli chromosome.

\section{Fluoroquinolones}

Plasmid-specified qnr elements cause an unusual mechanism of decreased fluoroquinolone susceptibility. Variants of the qnr element, which were first identified in K. pneumoniae,

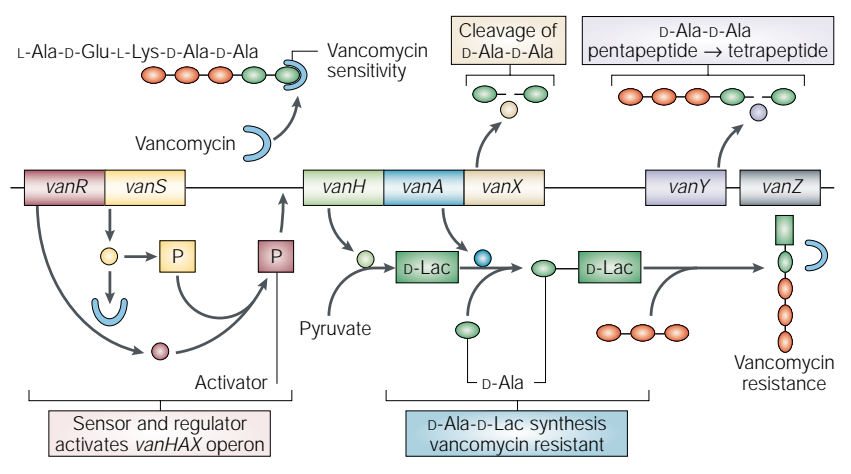

Figure 6. Vancomycin resistance gene system (VanA) in enterococci and Staphylococcus aureus. VanS: Membrane sensor encoder that controls the level of phosphorylation in the VanR protein. VanR: Encoder of the operon transcription factor of $\mathrm{VanH}, \mathrm{A}, \mathrm{X}$. VanH: Encoder of a dehydrogenase that can reduce pyruvate to D-Lac. VanA: Encoder of a ligase that catalyzes the formation of an ester bond between D-Ala and D-Lac. VanX: Encoder of a dipeptidase that hydrolyzes the D-Ala-D-Ala bond. VanY: Encoder of a carboxypeptidase which causes the hydrolysis of the D-Ala end bond of the carboxylate region and complements VanX. VanZ: Cause of teicoplanin resistance with an unspecified function. Reprinted by permission from Springer/Nature: Nature Reviews Genetics. See the following link for details: https://www.nature.com/articles/nrg1084. ${ }^{51}$

have been found in E. coli, Enterobacter cloacae, Providencia stuartii, Citrobacter freundii, Citrobacter koseri, Shigella flexneri 2b, and non-Typhi Salmonella enterica. The qnr gene, belonging to the family of proteins with pentapeptide repeats, likely induces resistance by protecting the inhibitory action of fluoroquinolones on DNA gyrase and IV topoisomerase. The mechanism of resistance to fluoroquinolone is led by another gene called $M f p A$, which has been detected in $M$. tuberculosis and contains a single type II topoisomerase. This protein is also a member of the family of proteins with pentapeptide repeats; furthermore, it acts as an inhibitor of DNA gyrase in M. tuberculosis by imitating the B-form DNA. The MfpA-DNA gyrase interaction is likely to interfere with the inhibitory function of compounds such as ciprofloxacin. The $q n r$ and $M f p A$ genes cause only low levels of resistance to fluoroquinolones by themselves, but they can increase such resistance when combined with other effective mechanisms.

\section{Efflux Systems and Porins}

Efflux pumps were first described as a kind of antibiotic resistance mechanisms for tetracyclines; now, however, they are described as a general and effective mechanism of resistance in many bacteria. Most of the proteins which produce the efflux pumps belong to five different families: the resistance-nodulation-division (RND), major facilitator (MF), small multidrug resistance (SMR), ATP-binding cassette $(\mathrm{ABC})$, and multidrug and toxic compound extrusion (MATE) (Figure 7). Efflux is driven forward by proteins in the RND, SMR, MF, and MATE families using the proton and sodium-motive force, and therefore is referred to as a secondary transport; however, the ATP hydrolysis drives the efflux forward on primary transporters (ABC). Efflux proteins also fall into two general categories. Some of them, such as tetracycline (Tet) and macrolide (Mef) transporters, are one-component systems that have a limited selection 


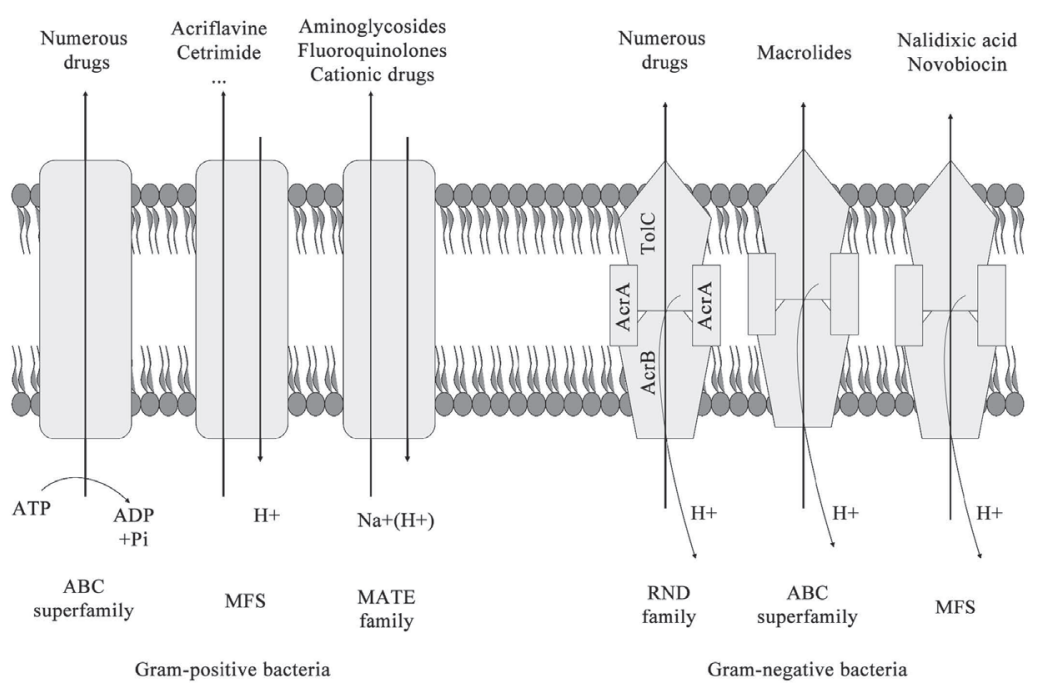

Figure 7. Different Classes of Efflux Pumps in Gram-Negative and Gram-Positive Bacteria. ${ }^{56}$

profile and act on few factors or many factors in the same drug class. The rest, like the members of the RND family, which need multiple structural proteins to develop resistance, have the ability to bind multiple drug combinations that are not structurally interrelated, and thus produce vast numbers of resistance phenotypes. The structure of RND-based efflux pumps that are found in a number of gram-negative bacteria allows them to transport the drug from the cytoplasm as well as the inner and outer membrane of the cellular coating. ${ }^{56}$

\section{Tetracyclines}

Today, more than 20 types of tetracycline-related efflux pump proteins have been reported. They are classified into six different groups. These proteins contain either 12 fragments (like TetA-E in gram-negative bacteria) or 14 fragments (such as TetK and TetL in gram-positive bacteria) which bridge through cell membranes. The expression of Class 1 proteins is controlled by a transcription suppressor such as TetR. The antibiotic inactivates the suppressor and allows the expression of tetracycline efflux pumps. In another method, the production of TetK and TetL by tetracycline can be induced by mechanisms that involve reducing the translation rate or its re-initiation. In general, the tetracycline efflux pump is not a protected similar system. ${ }^{57,58}$

\section{Macrolide-Lincosamide-Streptogramin Antibiotics}

MLS resistance is an $\mathrm{ABC}$ efflux protein that causes resistance to 14- and 15-membered macrolide antibiotics as well as streptogramin B in streptococci and staphylococci, but it does not affect clindamycin susceptibility. In staphylococci, the MsrA affiliates (VgaA and VgaB) are located on the plasmid. VgaA causes resistance to streptogramin A and lincosamides, and VGAB also develops the pristinamycin susceptibility (a combination of streptogramin A and B antibiotics). The Mef efflux transporters, which are the dominant macrolideresistance proteins in the United States, frequently and effectively act against 14- and 15-membered macrolides in Streptococcus; but strains expressing these proteins are susceptible to 16-membered macrolides, lincosamides, and streptogramin B. E. faecalis is normally resistant to quinupristin/dalfopristin, and this feature is attributed to the lsa gene, which encodes a streptogramin efflux protein belonging to the $\mathrm{ABC}$ family, since lsa mutation-induced inactivation causes quinupristin/dalfopristin susceptibility in this bacterium. Studies on efflux proteins have shown that eliminating an efflux system makes the bacteria susceptible to antibiotics even in the presence of chromosomal mutations reducing drug-binding affinity. ${ }^{59,60}$

\section{Fenicols}

Chloramphenicol and florfenicol are extended-spectrum antibiotics that are used in human clinical treatments (chloramphenicol) and animals (florfenicol). Considering the toxic effects (irreversible anemia), the current use of chloramphenicol is restricted to the treatment of some severe fatal infections such as bacterial meningitis in patients who are allergic to penicillin. Fenicol-specific efflux proteins have been reported in a number of important clinical bacteria and fall into eight different groups (E-1 to E-8). Overall, these proteins produce higher levels of resistance to multi-drug efflux proteins (referred to below), and members of the E-3 and E-4 groups produce resistance to both fenicols.

\section{Multi-Drug Resistance Efflux Systems}

In the past, it was thought that the envelope of gram-negative bacteria, as a strong inhibitor of drug penetration, affected antibiotic susceptibility. Later studies have shown, however, that most antibacterial agents effectively penetrate gramnegative bacteria, while they fail to achieve intracellular targets due to the presence of active efflux pumps in their membrane. The intrinsic resistance of gram-negative bacteria, such as E. coli, P. aeruginosa, Acinetobacter, Streptococcus mutans, Burkholderia cepacia, and Acinetobacter species, is attributed to the expression of RND efflux pumps. ${ }^{6}$ Tigecycline, approved by the FDA in 2005, has poor effectiveness against P. aeruginosa, Proteus mirabilis, Morganella morganii, and 
Klebsiella pneumonia which is attributed to RND systems. Studies have shown that the removal of ArcA orthologous in Morganella morganii, MexXY-OprM in P. aeruginosa, and ArcB orthologous in P. mirabilis increases the susceptibility to tigecycline by 16 to 133 times, while the removal of $\mathrm{ArcB}$ and ArcEF in E. coli exerts a more balanced effect (4 times) ${ }^{62-64}$ The bacillus multidrug resistance transporter $(\mathrm{Bmr})$ in Bacillus subtilis and Qac (quaternary ammonium compound) in S. aureus are $2 \mathrm{MDR}$ efflux proteins (major family members of MF) that were first detected and described in grampositive bacteria. Like many members of the RND family in gram-negative bacteria, Bmr is expressed continuously and thus provides intrinsic resistance to chloramphenicol and fluoroquinolones. Blt is another MDR efflux pump in Bacillus subtilis, which also contains spermidine in its list of target compounds. It is now thought that the natural function of Blt is to facilitate the removal of polyamines from the cell. The staphylococcal Qac systems provide resistance to antiseptics and disinfectants (e.g., quaternary ammonium compounds, chlorhexidine, and diamidines). Unlike most other MDR efflux proteins, these are specified on plasmids, a feature that facilitates their dissemination (Table 3). ${ }^{65-68}$

\section{Porins}

As mentioned previously, the outer membrane of the gramnegative cell envelope is a barrier to both hydrophobic and hydrophilic compounds. In order to circumvent this permeable barrier, these organisms have evolved porin proteins (e.g., OmpF in E. coli and OprD in $P$. aeruginosa) that function as "nonspecific" entry and exit points for antibiotics and other small-molecule organic chemicals. Imipenem (and to a lesser extent meropenem) and basic amino acids pass through OprD; mutations that decrease expression of the porin contribute to clinical imipenem resistance. Studies have shown that the expression of OprD and the MexEF-OprM efflux system is co-regulated, leading to the development of resistance to carbapenems and other
MexEF-OprM-dependent compounds in mutants where the expression of OprD and the efflux pump has been altered. In 1997, Hiramatsu et al identified a clinical S. aureus isolate in Japan that exhibited an intermediate level of resistance to vancomycin. Shortly thereafter, other bacteria with a glycopeptide-insensitive phenotype were identified in the United States. A prominent feature of the VISA isolates is the presence of a thickened cell wall. It is presumed that this property traps vancomycin and prevents the antibiotic from reaching its target. In 2006, the same researchers found reduced susceptibility to daptomycin in VISA samples. ${ }^{69,70}$ Mutations that produce resistance to polymyxin $\mathrm{B}$ in $P$. aeruginosa presumably involve changes in the bacterial cell envelope that do not involve porins. In Salmonella enterica serovar Typhimurium, PmrAB (a two-component regulatory system) regulates resistance to polymyxin by modifying lipopolysaccharide and lipid A. The RosAB efflux system of Yersinia enterocolitica also affects susceptibility to polymyxin B. ${ }^{71,72}$

\section{Conclusion}

More than half a century has passed since the first antibiotics were introduced commercially. It did not take long for microbes to promote their resistance systems, and the widespread use of many antibacterial drugs provided ideal conditions for the spread of MDR organisms. Most of the initial research focused on identifying ways to avoid the inhibitory effects of these drugs. Researchers such as Esther and Joshua Lederberg characterized the random nature of mutational events causing resistance to streptomycin. Other researchers like Tsutomu Watanabe thought that mutation alone would not be sufficient for explaining the MDR phenotype. These studies showed that resistance transfer factors (RTFs), later called resistance (R) factors and then plasmids, would provide the basis for multiple drug resistance caused by "infective heredity". For bacteria, there is more than one way to evade a drug class, and today, many bacteria

Table 3. Efflux Pump Systems Associated With Multi-Drug Resistance in Several Important Pathogens ${ }^{56}$

\begin{tabular}{|c|c|c|}
\hline Bacterial Organism & Efflux System & Representative Antibiotic Resistance \\
\hline P. aeroginosa & $\begin{array}{l}\text { MExAB-OprM } \\
\text { MexCD-OPrj } \\
\text { MexEF-OprN } \\
\text { MexHI-OPrD } \\
\text { Mexjk-oprM } \\
\text { MexVW-oprM } \\
\text { Mexy-OPrM }\end{array}$ & $\begin{array}{l}\text { BLA, and FQ } \\
4 \text { th gen ceph } \\
\text { FQ, Cm, Tmp, and Tri } \\
\text { EtBr, Nor, and Acr } \\
\text { Cip, Tet, and Erri } \\
\text { FQ, Cm, Tet, Ery, EtBr, and Acr } \\
\text { AG and Tig }\end{array}$ \\
\hline A. baumani & AdeABC & AG, FQ, TET, Ctx, Cm, Ery, and TMp \\
\hline S. maltophia & $\begin{array}{l}\text { smeABC } \\
\text { smeDEF }\end{array}$ & $\begin{array}{l}\text { AG, BLA, and FQ } \\
\text { MC, TET, FQ, CAR, Cm, and Ery }\end{array}$ \\
\hline A. серасіа & сеоАВ-орсМ & Cm, Cip, and Tmp \\
\hline B. pseudomallei & AmrAB-AprA & MAC and $A G$ \\
\hline E. coli & AcrAB-Tolc & FQ, BLA, TET, Cm, Acr, and Tri \\
\hline P. pneumoniae & AcrAB-Tolc & FQ, BLA, TET, Cip, Nor, EtBr, and TPP \\
\hline S. aureus & MepA & Tig, Mino, Tet, Cip, Nor, EtBr, and TPP \\
\hline E. faecalis & $\begin{array}{l}\text { EmeA } \\
\text { Lsa }\end{array}$ & $\begin{array}{l}\text { Nor, EtBr, Clind, Ery, and Nov } \\
\text { Clind and QD }\end{array}$ \\
\hline S. pneumoniae & pmrA & $\mathrm{FQ}, \mathrm{Acr}$, and $\mathrm{EtBr}$ \\
\hline
\end{tabular}


that continue to be unresponsive to all antimicrobial agents, even those they have not encountered previously, have been identified. Generally, bacteria adopt intricate strategies to avoid the lethal effects of antibiotics. Having an awareness of these mechanisms of resistance can help researchers design new drugs. As we face this critical problem, we need to be aware of and recognize the fluidity of the microbial genome and the ways in which resistance can appear by gene mutation or acquisition. Recognizing the potential for the emergence of resistance can give scientists a broader view for achieving deliberate discovery of novel compounds that will be needed to treat uncontrollable bacterial infections in the coming years. $^{73}$

\section{Authors' Contributions}

All authors contributed equally to this study.

\section{Conflict of Interest Disclosures}

The authors declare they have no conflicts of interest.

\section{References}

1. Amani J, Barjini KA, Moghaddam MM, Asadi A. In vitro synergistic effect of the CM11 antimicrobial peptide in combination with common antibiotics against clinical isolates of six species of multidrug-resistant pathogenic bacteria. Protein Pept Lett. 2015;22(10):940-951. doi:10.2174/0929866522666150728115 439

2. Moghaddam MM, Abolhassani F, Babavalian H, Mirnejad R, Azizi Barjini K, Amani J. Comparison of in vitro antibacterial activities of two cationic peptides CM15 and CM11 against five pathogenic bacteria: Pseudomonas aeruginosa, Staphylococcus aureus, Vibrio cholerae, Acinetobacter baumannii, and Escherichia coli. Probiotics Antimicrob Proteins. 2012;4(2):133-139. doi:10.1007/ s12602-012-9098-7

3. Moghaddam MM, Aghamollaei H, Kooshki H, Barjini KA, Mirnejad $\mathrm{R}$, Choopani A. The development of antimicrobial peptides as an approach to prevention of antibiotic resistance. Rev Med Microbiol. 2015;26(3):98-110.doi:10.1097/mrm.0000000000000032

4. Moghaddam MM, Barjini KA, Ramandi MF, Amani J. Investigation of the antibacterial activity of a short cationic peptide against multidrug-resistant Klebsiella pneumoniae and Salmonella typhimurium strains and its cytotoxicity on eukaryotic cells. World J Microbiol Biotechnol. 2014;30(5):1533-1540. doi:10.1007/ s11274-013-1575-y

5. Ventola CL. The antibiotic resistance crisis: part 1: causes and threats. P t. 2015;40(4):277-283.

6. Stokes HW, Gillings MR. Gene flow, mobile genetic elements and the recruitment of antibiotic resistance genes into Gramnegative pathogens. FEMS Microbiol Rev. 2011;35(5):790-819. doi:10.1111/j.1574-6976.2011.00273.x

7. Bennett PM. Plasmid encoded antibiotic resistance: acquisition and transfer of antibiotic resistance genes in bacteria. Br J Pharmacol. 2008;153 Suppl 1:S347-357. doi:10.1038/sj.bjp.0707607

8. Rowe-Magnus DA, Mazel D. The role of integrons in antibiotic resistance gene capture. Int J Med Microbiol. 2002;292(2):115125. doi:10.1078/1438-4221-00197

9. Heiat M, Rezaeimehr MR, Moghaddam MM, Ranjbar R, Najafi A. Molecular genetic analysis of quinolone resistance-determining region of DNA Gyrase-A in fluoroquinolones resistant Klebsiella pneumoniae based on GenBank data and reported studies. Mol Gen Microbiol Virol. 2014;29(4):211-215. doi:10.3103/ s0891416814040041

10. Hooper DC. Mechanisms of action and resistance of older and newer fluoroquinolones. Clin Infect Dis. 2000;31 Suppl 2:S24-28. doi:10.1086/314056
11. Robicsek A, Jacoby GA, Hooper DC. The worldwide emergence of plasmid-mediated quinolone resistance. Lancet Infect Dis. 2006;6(10):629-640. doi:10.1016/s1473-3099(06)70599-0

12. Rahimi H, Najafi A, Eslami H, Negahdari B, Moghaddam MM. Identification of novel bacterial DNA gyrase inhibitors: An in silico study. Res Pharm Sci. 2016;11(3):250-258.

13. Taniguchi $\mathrm{H}$, Aramaki $\mathrm{H}$, Nikaido $\mathrm{Y}$, et al. Rifampicin resistance and mutation of the rpoB gene in Mycobacterium tuberculosis. FEMS Microbiol Lett. 1996;144(1):103-108. doi:10.1111/j.1574-6968.1996.tb08515.x

14. Keshavjee S, Farmer PE. Tuberculosis, drug resistance, and the history of modern medicine. N Engl J Med. 2012;367(10):931936. doi:10.1056/NEJMra1205429

15. Grape M, Motakefi A, Pavuluri S, Kahlmeter G. Standard and real-time multiplex PCR methods for detection of trimethoprim resistance $\mathrm{dfr}$ genes in large collections of bacteria. Clin Microbiol Infect. 2007;13(11):1112-1118. doi:10.1111/j.1469$0691.2007 .01807 . x$

16. Skold O. Sulfonamide resistance: mechanisms and trends. Drug Resist Updat. 2000;3(3):155-160. doi:10.1054/drup.2000.0146

17. Capasso C, Supuran CT. Sulfa and trimethoprim-like drugs antimetabolites acting as carbonic anhydrase, dihydropteroate synthase and dihydrofolate reductase inhibitors. J Enzyme Inhib Med Chem. 2014;29(3):379-387. doi:10.3109/14756366.2013.7 87422

18. Alekshun MN, Levy SB. Molecular mechanisms of antibacterial multidrug resistance. Cell. 2007;128(6):1037-1050. doi:10.1016/j. cell.2007.03.004

19. Chopra I, Roberts M. Tetracycline antibiotics: mode of action, applications, molecular biology, and epidemiology of bacterial resistance. Microbiol Mol Biol Rev. 2001;65(2):232-260 ; second page, table of contents. doi:10.1128/mmbr.65.2.232-260.2001

20. De Stasio EA, Moazed D, Noller HF, Dahlberg AE. Mutations in 16S ribosomal RNA disrupt antibiotic--RNA interactions. EMBO J. 1989;8(4):1213-1216. doi:10.1002/j.1460-2075.1989.tb03494.x

21. Jana S, Deb JK. Molecular understanding of aminoglycoside action and resistance. Appl Microbiol Biotechnol. 2006;70(2):140-150. doi:10.1007/s00253-005-0279-0

22. Douthwaite S, Hansen LH, Mauvais P. Macrolide-ketolide inhibition of MLS-resistant ribosomes is improved by alternative drug interaction with domain II of 23S rRNA. Mol Microbiol. 2000;36(1):183-193. doi:10.1046/j.1365-2958.2000.01841.x

23. Long KS, Vester B. Resistance to linezolid caused by modifications at its binding site on the ribosome. Antimicrob Agents Chemother. 2012;56(2):603-612. doi:10.1128/aac.05702-11

24. Meka VG, Gold HS. Antimicrobial resistance to linezolid. Clin Infect Dis. 2004;39(7):1010-1015. doi:10.1086/423841

25. Gonzales RD, Schreckenberger PC, Graham MB, Kelkar S, DenBesten K, Quinn JP. Infections due to vancomycinresistant Enterococcus faecium resistant to linezolid. Lancet. 2001;357(9263):1179. doi:10.1016/s0140-6736(00)04376-2

26. Tian Y, Li T, Zhu Y, Wang B, Zou X, Li M. Mechanisms of linezolid resistance in staphylococci and enterococci isolated from two teaching hospitals in Shanghai, China. BMC Microbiol. 2014;14:292. doi:10.1186/s12866-014-0292-5

27. Bayer AS, Schneider T, Sahl HG. Mechanisms of daptomycin resistance in Staphylococcus aureus: role of the cell membrane and cell wall. Ann N Y Acad Sci. 2013;1277:139-158. doi:10.1111/ j.1749-6632.2012.06819.x

28. Silverman JA, Oliver N, Andrew T, Li T. Resistance studies with daptomycin. Antimicrob Agents Chemother. 2001;45(6):17991802. doi:10.1128/aac.45.6.1799-1802.2001

29. Steenbergen JN, Alder J, Thorne GM, Tally FP. Daptomycin: a lipopeptide antibiotic for the treatment of serious Gram-positive infections. J Antimicrob Chemother. 2005;55(3):283-288. doi:10.1093/jac/dkh546

30. Nicoloff H, Perreten V, McMurry LM, Levy SB. Role for tandem 
duplication and lon protease in AcrAB-TolC- dependent multiple antibiotic resistance (Mar) in an Escherichia coli mutant without mutations in marRAB or acrRAB. J Bacteriol. 2006;188(12):44134423. doi:10.1128/jb.01502-05

31. Nicoloff $\mathrm{H}$, Perreten $\mathrm{V}$, Levy SB. Increased genome instability in Escherichia coli lon mutants: relation to emergence of multipleantibiotic-resistant (Mar) mutants caused by insertion sequence elements and large tandem genomic amplifications. Antimicrob Agents Chemother. 2007;51(4):1293-1303. doi:10.1128/ aac.01128-06

32. Kumar S, Varela MF. Molecular mechanisms of bacterial resistance to antimicrobial agents. In: Mendez-Vilas A, ed. Microbial pathogens and strategies for combating them: science, technology and education. Formatex Research Center; 2013:522-534.

33. Wright GD. Bacterial resistance to antibiotics: enzymatic degradation and modification. Adv Drug Deliv Rev. 2005;57(10):1451-1470. doi:10.1016/j.addr.2005.04.002

34. Jacoby GA, Munoz-Price LS. The new beta-lactamases. N Engl J Med. 2005;352(4):380-391. doi:10.1056/NEJMra041359

35. Wilke MS, Lovering AL, Strynadka NC. Beta-lactam antibiotic resistance: a current structural perspective. Curr Opin Microbiol. 2005;8(5):525-533. doi:10.1016/j.mib.2005.08.016

36. Wright GD. Molecular mechanisms of antibiotic resistance. Chem Commun. 2011;47(14):4055-4061. doi:10.1039/C0CC05111J

37. Poirel L, Lambert T, Turkoglu S, Ronco E, Gaillard J, Nordmann P. Characterization of Class 1 integrons from Pseudomonas aeruginosa that contain the bla(VIM-2) carbapenem-hydrolyzing beta-lactamase gene and of two novel aminoglycoside resistance gene cassettes. Antimicrob Agents Chemother. 2001;45(2):546552. doi:10.1128/aac.45.2.546-552.2001

38. Doi Y, Arakawa Y. $16 \mathrm{~S}$ ribosomal RNA methylation: emerging resistance mechanism against aminoglycosides. Clin Infect Dis. 2007;45(1):88-94. doi:10.1086/518605

39. Kotra LP, Haddad J, Mobashery S. Aminoglycosides: perspectives on mechanisms of action and resistance and strategies to counter resistance. Antimicrob Agents Chemother. 2000;44(12):32493256. doi:10.1128/AAC.44.12.3249-3256.2000

40. Matsuoka M, Sasaki T. Inactivation of macrolides by producers and pathogens. Curr Drug Targets Infect Disord. 2004;4(3):217-240. doi:10.2174/1568005043340696

41. Wolter N, Smith AM, Farrell DJ, et al. Novel mechanism of resistance to oxazolidinones, macrolides, and chloramphenicol in ribosomal protein L4 of the pneumococcus. Antimicrob Agents Chemother. 2005;49(8):3554-3557. doi:10.1128/aac.49.8.35543557.2005

42. Schwarz S, Kehrenberg C, Doublet B, Cloeckaert A. Molecular basis of bacterial resistance to chloramphenicol and florfenicol. FEMS Microbiol Rev. 2004;28(5):519-542. doi:10.1016/j. femsre.2004.04.001

43. Moore IF, Hughes DW, Wright GD. Tigecycline is modified by the flavin-dependent monooxygenase TetX. Biochemistry. 2005;44(35):11829-11835. doi:10.1021/bi0506066

44. Volkers G, Palm GJ, Weiss MS, Wright GD, Hinrichs W. Structural basis for a new tetracycline resistance mechanism relying on the TetX monooxygenase. FEBS Lett. 2011;585(7):1061-1066. doi:10.1016/j.febslet.2011.03.012

45. Fuda CC, Fisher JF, Mobashery S. Beta-lactam resistance in Staphylococcus aureus: the adaptive resistance of a plastic genome. Cell Mol Life Sci. 2005;62(22):2617-2633. doi:10.1007/ s00018-005-5148-6

46. Chambers HF, Deleo FR. Waves of resistance: Staphylococcus aureus in the antibiotic era. Nat Rev Microbiol. 2009;7(9):629641. doi:10.1038/nrmicro2200

47. Courvalin P. Vancomycin resistance in gram-positive cocci. Clin Infect Dis. 2006;42 Suppl 1:S25-34. doi:10.1086/491711

48. Kruse $T$, Levisson $M$, de Vos WM, Smidt $H$. vanl: a novel D-Ala-D-Lac vancomycin resistance gene cluster found in Desulfitobacterium hafniense. Microb Biotechnol. 2014;7(5):456-
466. doi:10.1111/1751-7915.12139

49. Hong HJ, Hutchings MI, Buttner MJ. Vancomycin resistance VanS/ VanR two-component systems. In: Utsumi R, ed. Bacterial Signal Transduction: Networks and Drug Targets. New York, NY: Springer; 2008:200-213.

50. Perichon B, Courvalin P. VanA-type vancomycin-resistant Staphylococcus aureus. Antimicrob Agents Chemother. 2009;53(11):4580-4587. doi:10.1128/aac.00346-09

51. Hughes D. Exploiting genomics, genetics and chemistry to combat antibiotic resistance. Nat Rev Genet. 2003;4(6):432-441. doi:10.1038/nrg1084

52. Stanton TB, Humphrey SB. Isolation of tetracycline-resistant Megasphaera elsdenii strains with novel mosaic gene combinations of tet(O) and tet(W) from swine. Appl Environ Microbiol. 2003;69(7):3874-3882. doi:10.1128/aem.69.7.3874-3882.2003

53. Connell SR, Tracz DM, Nierhaus KH, Taylor DE. Ribosomal protection proteins and their mechanism of tetracycline resistance. Antimicrob Agents Chemother. 2003;47(12):3675-3681. doi:10.1128/aac.47.12.3675-3681.2003

54. Hisanaga T, Hoban DJ, Zhanel GG. Mechanisms of resistance to telithromycin in Streptococcus pneumoniae. J Antimicrob Chemother. 2005;56(3):447-450. doi:10.1093/jac/dki249

55. Zhanel GG, Dueck M, Hoban DJ, et al. Review of macrolides and ketolides: focus on respiratory tract infections. Drugs. 2001;61(4):443-498. doi:10.2165/00003495-200161040-00003

56. Soto SM. Role of efflux pumps in the antibiotic resistance of bacteria embedded in a biofilm. Virulence. 2013;4(3):223-229. doi:10.4161/viru.23724

57. Sapunaric FM, Aldema-Ramos M, McMurry LM. Tetracycline resistance: efflux, mutation, and other mechanisms. In White DG, Alekshun MN, McDermott PF, eds. Frontiers in Antimicrobial Resistance: A Tribute to Stuart B. Levy. Washington, DC: ASM Press; 2005:3-18.

58. Piddock LJ. Multidrug-resistance efflux pumps - not just for resistance. Nat Rev Microbiol. 2006;4(8):629-636. doi:10.1038/ nrmicro1464

59. Chesneau O, Ligeret H, Hosan-Aghaie N, Morvan A, Dassa E. Molecular analysis of resistance to streptogramin A compounds conferred by the Vga proteins of staphylococci. Antimicrob Agents Chemother. 2005;49(3):973-980. doi:10.1128/aac.49.3.973980.2005

60. Li XZ, Nikaido H. Efflux-mediated drug resistance in bacteria: an update. Drugs. 2009;69(12):1555-1623. doi:10.2165/11317030000000000-00000

61. Li XZ, Plesiat P, Nikaido $H$. The challenge of efflux-mediated antibiotic resistance in Gram-negative bacteria. Clin Microbiol Rev. 2015;28(2):337-418. doi:10.1128/cmr.00117-14

62. Ochs MM, McCusker MP, Bains M, Hancock RE. Negative regulation of the Pseudomonas aeruginosa outer membrane porin OprD selective for imipenem and basic amino acids. Antimicrob Agents Chemother. 1999;43(5):1085-1090. doi:10.1128/ aac.43.5.1085

63. Hocquet D, Nordmann P, El Garch F, Cabanne L, Plesiat P. Involvement of the MexXY-OprM efflux system in emergence of cefepime resistance in clinical strains of Pseudomonas aeruginosa. Antimicrob Agents Chemother. 2006;50(4):13471351. doi:10.1128/aac.50.4.1347-1351.2006

64. Rodriguez-Martinez JM, Poirel L, Nordmann P. Molecular epidemiology and mechanisms of carbapenem resistance in Pseudomonas aeruginosa. Antimicrob Agents Chemother. 2009;53(11):4783-4788. doi:10.1128/aac.00574-09

65. Degregorio D, Sadeghi SJ, Di Nardo G, Gilardi G, Solinas SP. Understanding uncoupling in the multiredox centre P450 3A4BMR model system. J Biol Inorg Chem. 2011;16(1):109-116. doi:10.1007/s00775-010-0708-0

66. Klyachko KA, Schuldiner S, Neyfakh AA. Mutations affecting substrate specificity of the Bacillus subtilis multidrug transporter Bmr. J Bacteriol. 1997;179(7):2189-2193. doi:10.1128/ 
jb.179.7.2189-2193.1997

67. Steinfels E, Orelle C, Fantino JR, et al. Characterization of YvcC $(\mathrm{BmrA})$, a multidrug $\mathrm{ABC}$ transporter constitutively expressed in Bacillus subtilis. Biochemistry. 2004;43(23):7491-7502. doi:10.1021/bi0362018

68. Fraise AP. Susceptibility of antibiotic-resistant cocci to biocides. J Appl Microbiol. 2002;92 Suppl:158s-162s. doi:10.1046/j.13652672.92.5s1.2.x

69. Hiramatsu K, Aritaka N, Hanaki H, et al. Dissemination in Japanese hospitals of strains of Staphylococcus aureus heterogeneously resistant to vancomycin. Lancet. 1997;350(9092):1670-1673. doi:10.1016/s0140-6736(97)07324-8

70. Cui L, Tominaga E, Neoh HM, Hiramatsu K. Correlation between Reduced Daptomycin Susceptibility and Vancomycin Resistance in Vancomycin-Intermediate Staphylococcus aureus. Antimicrob Agents Chemother. 2006;50(3):1079-1082. doi:10.1128/ aac.50.3.1079-1082.2006

71. Bolla JM, Alibert-Franco S, Handzlik J, et al. Strategies for bypassing the membrane barrier in multidrug resistant Gram-negative bacteria. FEBS Lett. 2011;585(11):1682-1690. doi:10.1016/j. febslet.2011.04.054

72. Delcour $\mathrm{AH}$. Outer membrane permeability and antibiotic resistance. Biochim Biophys Acta. 2009;1794(5):808-816. doi:10.1016/j.bbapap.2008.11.005

73. Ling LL, Schneider T, Peoples AJ, et al. A new antibiotic kills pathogens without detectable resistance. Nature. 2015;517(7535):455459. doi:10.1038/nature14098 